\title{
VALIDAÇÃO DE MÉTODO MULTIRRESÍDUO PARA DETERMINAÇÃO DE PESTICIDAS EM ALIMENTOS EMPREGANDO QuEChERS E UPLC-MS/MS
}

\author{
Sonia C. N. Queiroz*, Vera L. Ferracini e Maria A. Rosa \\ Embrapa Meio Ambiente, CP 69, 13820-000 Jaguariúna - SP, Brasil
}

Recebido em 1/2/11; aceito em 23/5/11; publicado na web em 22/7/11

\begin{abstract}
MULTIRESIDUE METHOD VALIDATION FOR DETERMINATION OF PESTICIDES IN FOOD USING QuEChERS AND UPLCMS/MS. This paper presents a practical and rapid method which was validated for simultaneous quantification and confirmation of 29 pesticides in fruits and vegetables using ultra performance liquid chromatography-tandem mass spectrometry (UPLC-MS/MS). The samples were extracted following the method known as QuEChERS. Using the developed chromatographic conditions, the pesticides can be separated in less than 9 min. Two multiple reaction monitoring (MRM) assays were used for each pesticide. Four representative matrices (lettuce, tomato, apple and grapes) were selected to investigate the effect in recoveries and precision. Typical recoveries ranged from $70-120 \%$, with relative standard deviation (RSDs) lower than $20 \%$.
\end{abstract}

Keywords: pesticides; QuEChERS; UPLC-MS/MS.

\section{INTRODUÇÃO}

Nos últimos anos tem havido uma preocupação crescente com relação à presença de resíduos de pesticidas nos alimentos, devido aos possíveis efeitos adversos à saúde dos seres humanos se expostos por longos períodos, especialmente em crianças que consomem maiores porções de frutas e hortaliças em relação à massa corporal quando comparados aos adultos e são mais susceptíveis às substâncias químicas tóxicas por estarem nos estágios iniciais de desenvolvimento. ${ }^{1}$

Visando proteger a saúde dos consumidores agências governamentais, como a Agência Nacional de Vigilância Sanitária (ANVISA) no Brasil, ${ }^{2}$ limitam as quantidades de pesticidas em alimentos e estabelecem limites máximos de resíduos (LMRs) para as diversas combinações de pesticidas e commodities.

Historicamente, a cromatografia gasosa (GC) tem sido a técnica selecionada para a análise multirresíduo de pesticidas em produtos de origem vegetal. ${ }^{3,4}$ Entretanto, muitos pesticidas modernos são polares ou iônicos, os quais apresentam menor persistência e toxicidade ao meio ambiente, e não podem ser analisados diretamente por GC. A cromatografia líquida de alta eficiência acoplada à espectrometria de massas em série (LC-MS/MS) é a melhor escolha para substâncias que apresentam volatilidade baixa e/ou instabilidade térmica. A cromatografia líquida é muito efetiva na separação dos analitos, enquanto que a espectrometria de massas em série permite a sua identificação e/ou confirmação em concentrações da ordem de $\mu \mathrm{g} \mathrm{kg}^{-1}$ ou menores..$^{5-7}$

Embora as técnicas cromatográficas acopladas à espectrometria de massas sejam muito sensíveis e seletivas, deve ser realizada uma etapa de preparo da amostra. Atualmente, um dos métodos de preparo de amostra para análise de resíduos mais citados na literatura ${ }^{8}$ é o descrito por Anastassiades e colaboradores, ${ }^{9}$ conhecido como QuEChERS (Quick, Easy, Cheap, Effective, Robust and Safe). Vários laboratórios no mundo têm implementado o método QuEChERS devido às vantagens que oferece em relação aos convencionais, conforme o próprio nome diz..$^{5,8-10}$

A etapa de separação cromatográfica pode ser demorada quando é analisado um número grande de compostos. Na tentativa de contornar este problema várias estratégias têm sido adotadas, dentre elas,

*e-mail: sonia@cnpma.embrapa.br o uso de colunas recheadas com partículas de tamanho inferior a 2 $\mu \mathrm{m}$, o qual é denominado cromatografia líquida de ultraeficiência (ultra performance liquid chromatography-UPLC). ${ }^{11,12}$ Esta técnica tem se tornado uma tendência mundial, haja vista que tem como vantagem principal o aumento expressivo na eficiência de separação dos analitos, mesmo com vazões elevadas da fase móvel. ${ }^{13,14}$ Como resultado, não somente há o aumento da eficiência, mas também se obtém uma melhor resolução, um menor tempo de análise e uma melhor detectabilidade. A combinação de UPLC-MS/MS fornece vantagens significativas com relação à seletividade, detectabilidade e velocidade de análise. Até o momento, a UPLC tem sido aplicada principalmente no campo farmacêutico, ${ }^{15-17}$ havendo uma carência de dados na literatura sobre a determinação de resíduos de pesticidas em amostras de alimentos. ${ }^{18-20}$ Alguns exemplos de aplicação da UPLC-MS/MS em análises de resíduos de pesticidas em alimentos foram publicados recentemente para as matrizes citros, ${ }^{21}$ chá, ${ }^{22}$ frutas e alimentos infantis ${ }^{23}$ e vinho. ${ }^{24}$

Nesse estudo é apresentada a validação de um método rápido para a determinação multirresíduo de pesticidas em alface, tomate, maçã e uva, empregando para extração o método QuEChERS seguido de UPLC-MS/MS.

Para a seleção das matrizes levou-se em consideração commodities classificadas dentro do grupo de alto risco em relação à exposição aos pesticidas, por ser necessário grande número pulverizações durante o cultivo. ${ }^{25,26}$ Os pesticidas (Figura 1) foram escolhidos com base no documento da ANVISA, no qual são sugeridos os grupos de pesticidas a serem monitorados por métodos multirresíduo em frutas, legumes e verduras ${ }^{27}$ e também no levantamento dos produtos aplicados no campo. ${ }^{2}$

\section{PARTE EXPERIMENTAL}

\section{Reagentes}

Todos os reagentes utilizados foram grau HPLC ou analítico, com pureza entre 99,05 a 99,9\%. Os solventes orgânicos foram acetonitrila e metanol, grau HPLC (Tédia, EUA) e os reagentes sulfato de magnésio anidro P.A. (J. T. Baker, Japão); cloreto de sódio P.A. (Spectrum, EUA); citrato de trissódio di-hidratado P.A. (J. T. Baker, Áustria); hidrogeno citrato de dissódio sesqui-hidratado P.A. (Aldri- 
<smiles>c1ccc2[nH]c(-c3cscn3)nc2c1</smiles>

1<smiles>CNc1nc(=O)n(C2CCCCC2)c(=O)n1C</smiles>

2<smiles>CCNc1nc(Cl)nc(NC(C)C)n1</smiles>

3<smiles>COC(=O)c1nc(N(C)C)nc(C)c1C</smiles>

4<smiles>CC1COC(Cn2cncn2)(c2ccc(Oc3ccc(Cl)cc3)cc2Cl)O1</smiles>

7<smiles>CNC(=O)Oc1cccc2c1OC(C)(C)C2</smiles>

10

II $\left(\mathrm{CH}_{3} \mathrm{O}\right)_{2} \mathrm{PSCHCH} \mathrm{CO}_{2} \mathrm{CH}_{2} \mathrm{CH}_{3}$ $\mathrm{CO}_{2} \mathrm{CH}_{2} \mathrm{CH}_{3}$

11<smiles>Cc1cccc(C)c1N(C(=O)Cc1ccccc1)C(C)C=O</smiles>
$\circ$<smiles>COC(=O)C=C(C)OP(=O)=O</smiles>

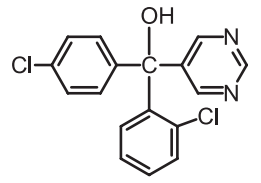

15<smiles>COCC(=O)N(c1c(C)cccc1C)C(C)C(C)=O</smiles>

5<smiles>C=CCOC(Cn1ccnc1)c1ccc(Cl)cc1Cl</smiles><smiles>CC1(C)CON(Cc2ccccc2Cl)C1=O</smiles>

13<smiles>CO/C=C(/CO)c1ccccc1Oc1cc(Oc2ccccc2C#N)ncn1</smiles>

16<smiles>CNC(=O)CSP(C)OC</smiles>

18<smiles>Cc1nnc(C(C)(C)C)c(=O)n1N</smiles>

22<smiles>CC(=O)Nc1ccc(Cl)c(Cl)c1</smiles>

26<smiles>CCNc1nc(Cl)nc(NCC)n1</smiles>

19<smiles>CNC(=O)ON=CC(C)(C)SC</smiles>

23<smiles>COP(=S)(OC)Oc1ccc(SC)c(C)c1</smiles>

27<smiles>CC(C1CC1)C(O)(Cn1cncn1)c1ccc(Cl)cc1</smiles>

20<smiles>O=[N+]([O-])/N=C1\NCCN1CCc1ccc(Cl)nc1</smiles>

24<smiles>CC1(C)C2(C)C(=O)N(c3cc(Cl)cc(Cl)c3)C(=O)C12C</smiles>

28<smiles>CNC(=O)/C=C(\C)O[PH](C)=O</smiles>

21<smiles>CCC(C)n1c(=O)[nH]c(C)c(Br)c1=O</smiles>

25<smiles>N#Cc1c[nH]cc1-c1cccc2c1OC(F)(F)O2</smiles>

29

Figura 1. Estruturas dos pesticidas. 1) tiabendazol, 2) hexazinone, 3) atrazina, 4) pirimicarbe, 5) metalaxil, 6) diazinona, 7) benalaxil, 8) difenoconazol, 9) imazalil, 10) carbofurano, 11) cis-mevinfós, 12) clomazona, 13) tebuconazol, 14) malationa, 15) fenarimol, 16) azoxistrobina, 17) diclorvós, 18) dimetoato, 19) simazina, 20) ciproconazol, 21) monocrotofós, 22) metribuzin, 23) aldicarbe, 24) imidacloprido, 25) bromacila, 26) diuron, 27) fentiona, 28) procimidona, 29) fludioxonil

ch, Alemanha); Bondesil-PSA, $40 \mu \mathrm{m}$ (Varian, EUA). Os seguintes padrões de pesticidas foram utilizados: tiabendazol $(98,5 \%)$, hexazinona $(98,0 \%)$, metalalaxil $(99,5 \%)$, difenoconazol $(99,5 \%)$, imazalil $(96,0 \%)$, clomazona $(93,0 \%)$, tebuconazol $(98,5 \%)$, azoxistrobina $(99,0 \%)$, dimetoato $(99,0 \%)$, simazina $(99,5 \%)$, metribuzin $(99,0 \%)$, aldicarbe (98,5\%), imidacloprido $(98,0 \%)$, diuron $(97,7 \%)$, fentiona $(96,5 \%)$ e procimidona $(99,5 \%)$ adquiridos da empresa Dr. Ehrenstorfer (Augsburg, Alemanha); atrazina (97,5\%) obtida da empresa
Fluka (St. Louis, EUA); pirimicarbe $(99,0 \%)$, diazinona $(99,0 \%)$, benalaxil $(99,9 \%)$, carbofurano $(99,9 \%)$, cis-mevinfós $(98,6 \%)$, fenarimol $(99,8 \%)$, diclorvós $(98,8 \%)$, fludioxonil $(99,9 \%)$ da empresa Riedel-de-Haen (Zwijndrecht, Alemanha); malationa $(99,4 \%)$, ciproconazol $(98,0 \%)$, monocrotofós $(99,5 \%)$ e bromacila $(98,6 \%)$ foram adquiridos da empresa ChemService (West Chester, EUA). A água ultrapurificada foi obtida de um sistema Milli-Q Simplicity 185 (Millipore, Beldford, EUA). 


\section{Equipamentos}

\section{Cromatógrafo de ultraeficiência acoplado ao espectrômetro de massas}

Foi utilizado um cromatógrafo de ultra-altaeficiência acoplado a um espectrômetro de massas tipo triploquadrupolo e interface por eletronebulização (UPLC-ESI-MS/MS) modelo Quattro Premier XE, Waters (Milford, MA, EUA)

Inicialmente foram feitas as infusões dos padrões individuais dos pesticidas no espectrômetro de massas a fim de otimizar as respostas dos íons precursores. Foram monitoradas as respostas de dois íons produto, modo MRM (multiple reaction monitoring), sendo que o mais abundante foi selecionado para quantificação e o menos abundante para confirmação. Os fragmentos selecionados para determinação em modo MRM para os íons precursores estão apresentados na Tabela 1. O equipamento foi operado em modo MRM com ionização por eletronebulização, no modo íon positivo e negativo. A voltagem do capilar foi otimizada a 4,0 KV. Nitrogênio foi usado como gás de dessolvatação à $400{ }^{\circ} \mathrm{C}$ com fluxo de $500 \mathrm{~L} \mathrm{~h}^{-1}$, argônio foi usado como gás de colisão com fluxo de $20 \mathrm{~mL} \mathrm{~min}^{-1}$.

Foi utilizada uma coluna Acquity UPLC ${ }^{\circledR}$ BEH C-18 $(1,7 \mu \mathrm{m}$, $2,1 \mathrm{~mm}$ d.i. $\mathrm{x} 100 \mathrm{~mm}$ ), temperatura de $40{ }^{\circ} \mathrm{C}$ e o seguinte gradiente de eluição: inicialmente com $45 \%$ (v/v) das fases A (Metanol) e B (solução aquosa contendo $0,1 \%$ ácido fórmico), com rampa linear até atingir $90 \%$ da fase $\mathrm{A}$ em 8,0 min; em seguida, retorna a $45 \%$ de $\mathrm{A}$ em 8,5 min permanecendo nesta composição até 9,0 min, totalizando o tempo de análise de 9,0 min. A vazão da fase móvel foi mantida a $0,3 \mathrm{~mL} \mathrm{m^{-1 }}$ e o volume de injeção foi de $10 \mu \mathrm{L}$.

\section{Preparo das soluções padrão dos pesticidas}

Solução intermediária de 29 analitos foi preparada em $\mathrm{MeOH}$ HPLC na concentração de $10 \mu \mathrm{g} \mathrm{mL}^{-1}$, a partir de solução estoque de $1000 \mu \mathrm{g} \mathrm{mL}^{-1}$ de cada analito. As soluções usadas para a construção das curvas analíticas foram obtidas em concentrações entre 0,100 a $0,001 \mu \mathrm{g} \mathrm{mL}^{-1} \mathrm{em} \mathrm{CH} \mathrm{CH}_{3} \mathrm{OH} / \mathrm{H}_{2} \mathrm{O}$ com $0,1 \%$ ácido fórmico (50\%, v/v), após diluições adequadas da solução intermediária de $10 \mu \mathrm{g} \mathrm{mL} \mathrm{mL}^{-1}$. As soluções de trabalho foram preparadas diariamente. A balança utilizada para as pesagens dos padrões bem como as vidrarias usadas no preparo das soluções foram calibradas por empresas credenciadas junto ao Inmetro - Rede Brasileira de Calibração (RBC) e em conformidade com os requisitos da NBR ISO/IEC 17025.

\section{Obtenção das amostras}

As amostras de alface e tomate foram cultivadas no campo experimental da Embrapa Meio Ambiente sem o uso de pesticidas e as amostras de maçã e uva foram adquiridas em estabelecimentos comerciais, analisadas e após a verificação da ausência de pesticidas foram utilizadas na validação como amostras branco.

Tabela 1. Dados de aquisição usados nas análises de 29 pesticidas por UPLC-MS/MS

\begin{tabular}{|c|c|c|c|c|c|c|c|c|c|c|}
\hline $\mathrm{N}^{\circ}$ & Composto & $\begin{array}{c}\mathrm{t}_{\mathrm{R}} \\
(\mathrm{min})\end{array}$ & $\begin{array}{c}\text { Modo } \\
\text { ionização }\end{array}$ & $m / z$ & $\begin{array}{c}\text { Transição } 1 \\
\text { Massas }(m / z)\end{array}$ & $\begin{array}{l}\text { E. C. } \\
\text { (V) }\end{array}$ & $\begin{array}{l}\text { E. Col. }{ }^{b} \\
\text { (V) }\end{array}$ & $\begin{array}{c}\text { Transição } 2 \\
\text { (confirmação) }\end{array}$ & $\begin{array}{l}\text { E. C. } \\
\text { (V) }\end{array}$ & $\begin{array}{c}\text { E. Col. }{ }^{\text {b }} \\
\text { (V) }\end{array}$ \\
\hline 1 & Tiabendazol & 1,13 & + & 201,68 & 174,60 & 49,0 & 24,0 & 130,70 & 49,0 & 30,0 \\
\hline 2 & Hexazinona & 2,69 & + & 252,87 & 170,80 & 29,0 & 16,0 & 70,70 & 29,0 & 34,0 \\
\hline 3 & Atrazina & 3,57 & + & 215,73 & 173,60 & 35,0 & 14,0 & 95,60 & 35,0 & 24,0 \\
\hline 4 & Pirimicarbe & 1,72 & + & 238,84 & 71,70 & 28,0 & 22,0 & 181,70 & 28,0 & 16,0 \\
\hline 5 & Metalaxil & 3,72 & + & 279,77 & 219,80 & 23,0 & 14,0 & 191,80 & 23,0 & 18,0 \\
\hline 6 & Diazinona & 6,36 & + & 304,85 & 168,70 & 36,0 & 20,0 & 152,70 & 36,0 & 22,0 \\
\hline 7 & Benalaxil & 6,36 & + & 326,02 & 147,70 & 25,0 & 20,0 & 90,70 & 25,0 & 40,0 \\
\hline 8 & Difenoconazol & 7,04 & + & 405,98 & 250,70 & 42,0 & 26,0 & 187,60 & 42,0 & 42,0 \\
\hline 9 & Imazalil & 3,07 & + & 296,91 & 254,70 & 37,0 & 18,0 & 158,60 & 37,0 & 22,0 \\
\hline 10 & Carbofurano & 2,65 & + & 221,78 & 164,60 & 21,0 & 12,0 & 122,60 & 21,0 & 24,0 \\
\hline 11 & cis-Mevinfós & 1,72 & + & 224,67 & 126,60 & 13,0 & 14,0 & 192,60 & 13,0 & 10,0 \\
\hline 12 & Clomazona & 4,22 & + & 239,97 & 124,60 & 24,0 & 18,0 & 88,70 & 24,0 & 38,0 \\
\hline 13 & Tebuconazol & 6,35 & + & 307,85 & 124,60 & 32,0 & 42,0 & 150,60 & 32,0 & 24,0 \\
\hline 14 & Malationa & 4,97 & + & 330,68 & 126,50 & 18,0 & 12,0 & 98,70 & 18,0 & 22,0 \\
\hline 15 & Fenarimol & 5,59 & + & 330,82 & 267,70 & 44,0 & 24,0 & 80,60 & 44,0 & 28,0 \\
\hline 16 & Azoxistrobina & 4,47 & + & 404,08 & 371,80 & 23,0 & 12,0 & 328,80 & 23,0 & 30,0 \\
\hline 17 & Diclorvos & 2,61 & + & 220,66 & 108,60 & 32,0 & 18,0 & 78,70 & 32,0 & 24,0 \\
\hline 18 & Dimetoato & 1,51 & + & 229,51 & 198,50 & 16,0 & 10,0 & 124,60 & 16,0 & 16,0 \\
\hline 19 & Simazina & 2,64 & + & 201,75 & 131,60 & 37,0 & 18,0 & 123,70 & 37,0 & 16,0 \\
\hline 20 & Ciproconazol & 5,11 & + & 291,84 & 124,74 & 29,0 & 26,0 & 69,87 & 29,0 & 18,0 \\
\hline 21 & Monocrotofós & 1,08 & + & 223,63 & 126,50 & 18,0 & 14,0 & 97,50 & 18,0 & 14,0 \\
\hline 22 & Metribuzin & 2,68 & + & 214,67 & 186,60 & 29,0 & 16,0 & 82,40 & 29,0 & 16,0 \\
\hline 23 & Aldicarbe & 2,07 & + & 212,62 & 88,60 & 24,0 & 12,0 & 115,60 & 24,0 & 10,0 \\
\hline 24 & Imidacloprido & 1,26 & + & 255,80 & 174,8 & 23,0 & 21,0 & 208,80 & 23,0 & 21,0 \\
\hline 25 & Bromacila & 2,68 & + & 260,67 & 204,60 & 18,0 & 14,0 & 187,60 & 18,0 & 30,0 \\
\hline 26 & Diuron & 3,93 & + & 232,67 & 71,70 & 30,0 & 24,0 & 159,50 & 30,0 & 32,0 \\
\hline 27 & Fentiona & 6,31 & + & 278,74 & 168,50 & 27,0 & 14,0 & 246,60 & 27,0 & 14,0 \\
\hline 28 & Procimidona & 5,52 & + & 283,75 & 255,70 & 38,0 & 18,0 & 94,70 & 38,0 & 22,0 \\
\hline 29 & Fludioxonil & 4,92 & + & 248,72 & 228,60 & 11,0 & 10,0 & 157,60 & 11,0 & 26,0 \\
\hline
\end{tabular}

${ }^{\mathrm{a}}$ E.C. $=$ Energia do cone; ${ }^{\mathrm{b}}$ E. Col. $=$ Energia de colisão. 


\section{Fortificação das amostras}

As amostras branco foram fortificadas nas concentrações 0,005 e $0,010 \mathrm{mg} \mathrm{kg}^{-1}$ para estudo de exatidão e precisão do método. As concentrações foram selecionadas de forma a atender o menor $\operatorname{LMR}\left(0,010 \mathrm{mg} \mathrm{kg}^{-1}\right)$, estabelecido para pesticidas não registrados e/ou proibidos, e devido à alta sensibilidade do equipamento foi possível realizar um nível abaixo $\left(0,005 \mathrm{mg} \mathrm{kg}^{-1}\right)$. As fortificações foram realizadas com adição de volumes adequados de uma solução intermediária de concentração $1,000 \mu \mathrm{g} \mathrm{mL}^{-1}$, preparada em $\mathrm{MeOH}$, e deixadas em repouso por $30 \mathrm{~min}$ antes das análises.

\section{Preparação das amostras}

As amostras de tomate, alface, maçã e uva foram homogeneizadas em um um blixer 3 da marca Robout Coupe. Após a homogeneização, os pesticidas foram extraídos utilizando-se o método QuEChERS. Um total de $10 \mathrm{~g}$ da amostra foi pesado em um tubo de centrífuga de politetrafluoretileno $(50 \mathrm{~mL})$ e após a adição de $10 \mathrm{~mL}$ de acetonitrila os tubos foram agitados em vortex (Minishaker IKA $1400 \mathrm{rpm}$ ) por 1 min. Em seguida, foram adicionados 4,0 g sulfato de magnésio anidro; $1,0 \mathrm{~g}$ de cloreto de sódio; $1,0 \mathrm{~g}$ de citrato de trissódio di-hidratado e $0,5 \mathrm{~g}$ de hidrogenocitrato de dissódio sesqui-hidratado. Após a agitação por $1 \mathrm{~min}$, a amostra foi sonicada no ultrassom (Transsonic 460/H) por $20 \mathrm{~min}$, com gelo e, em seguida, centrifugada (Thermo Scientific Heraeus-Multifuge 3L-R) por 5 min a $10.000 \mathrm{rpm}$, a 10 ${ }^{\circ} \mathrm{C}$. Cinco $\mathrm{mL}$ do sobrenadante foram transferidos para o tubo de centrífuga contendo $125 \mathrm{mg}$ de Bondesil-PSA e $750 \mathrm{mg}$ de sulfato de magnésio. Após a agitação da mistura por $30 \mathrm{~s}$ e centrifugação por 5 min a $10.000 \mathrm{rpm}$ e $10^{\circ} \mathrm{C}$, uma alíquota de $2 \mathrm{~mL}$ do sobrenadante foi evaporada até a secura, em banho de gelo. O resíduo foi ressuspenso em $2 \mathrm{~mL}$ de fase móvel $\mathrm{CH}_{3} \mathrm{OH} / \mathrm{H}_{2} \mathrm{O}$ com $0,1 \%$ ácido fórmico $(50 \%$, $\mathrm{v} / \mathrm{v}$ ), sonicado por $3 \mathrm{~min}$, filtrado em filtro Millex HV (Durapore) com tamanho de poro $0,45 \mu \mathrm{m}$ e $13 \mathrm{~mm}$ de diâmetro com corpo em polietileno da Millipore, diretamente para o vial para posterior injeção de $10 \mu \mathrm{L}$ no sistema UPLC-MS/MS.

\section{Quantificação dos pesticidas}

Para quantificação dos pesticidas no UPLC-MS/MS, tanto nos estudos de recuperação quanto nas análises de amostras comerciais, foram construídas curvas analíticas na matriz com cinco níveis de concentração: $1 ; 5 ; 10 ; 50 ; 100 \mathrm{ng} \mathrm{mL}^{-1}$, que foram injetados em duplicata $(\mathrm{n}=2)$. Os padrões dos pesticidas foram adicionados aos extratos secos da matriz branco e o volume completado para $2 \mathrm{~mL}$ com $\mathrm{CH}_{3} \mathrm{OH} / \mathrm{H}_{2} \mathrm{O}$ com $0,1 \%$ ácido fórmico $(50 \%, \mathrm{v} / \mathrm{v})$. O uso de curva na matriz foi selecionado para evitar efeitos matriz que causam enriquecimento ou supressão do sinal obtido pelo espectrômetro de massas.

\section{RESULTADOS E DISCUSSÃO}

As matrizes selecionadas para este trabalho foram alface, tomate, maçã e uva. De acordo com a classificação da ANVISA alface é a espécie representativa do grupo que possui alta taxa de água e clorofila. Esta matriz faz parte da classe de vegetais folhosos. O tomate, a maçã e a uva são espécies representativas de outro grupo com alta taxa de água e pouca ou nenhuma taxa de clorofila. A maçã faz parte da classe dos pomos, a uva das frutas pequenas e o tomate das hortaliças/frutos. ${ }^{27}$ Segundo a classificação do SANCO a uva pertence ao grupo de commodities com alto teor de ácido. ${ }^{28}$

Vinte e nove pesticidas de diferentes classes foram avaliados. A seleção das substâncias foi realizada com base no documento da ANVISA, ${ }^{27}$ onde constam os pesticidas que devem ser monitorados através de métodos multirresíduo e, também, pelo histórico de uso pelos agricultores.

\section{Preparação da amostra}

A estratégia utilizada na escolha do método de preparo de amostra influencia diretamente o desempenho global do método analítico. Para este trabalho, o QuEChERS, ${ }^{29}$ que é o procedimento de extração de resíduos de pesticidas selecionado para ser o oficial pela Comunidade Europeia, foi escolhido devido às vantagens que oferece como rapidez, facilidade, baixo custo, eficiência, robustez e segurança.

\section{Otimização das condições do espectrômetro de massas e UPLC}

As condições de operação do espectrômetro de massas (voltagens do cone e de colisão) foram otimizadas em modo MRM, através da infusão de cada solução contendo $1 \mu \mathrm{g} \mathrm{mL}^{-1}$ do pesticida em $\mathrm{CH}_{3} \mathrm{OH} /$ $\mathrm{H}_{2} \mathrm{O}$ com $0,1 \%$ ácido fórmico (50\%, v/v), sob uma vazão de $20 \mu \mathrm{L}$ $\min ^{-1}$. Os dados de aquisição otimizados estão mostrados na Tabela 1. As análises quantitativa e confirmatória foram realizadas em modo MRM, através do monitoramento de duas transições de $\mathrm{m} / \mathrm{z}$ para o íon precursor, sendo que a que gerou sinal mais intenso foi utilizada para quantificação e a menos intensa para confirmação. O critério de confirmação atende o recomendado pela Comunidade Europeia ${ }^{28}$ que é de quatro pontos de identificação, correspondentes à seleção de um íon precursor e dois íons produtos (duas transições de $m / z$ ), além do tempo de retenção na coluna.

Após a otimização do modo MRM via infusão, foram otimizadas as condições cromatográficas. Para isso foram testados vários programas de gradiente utilizando como fase móvel metanol e solução aquosa contendo $0,1 \%$ de ácido fórmico, sob uma vazão de $0,3 \mathrm{~mL}$ $\min ^{-1}$, e foram avaliadas as separações obtidas para os pesticidas. As melhores condições cromatográficas estão descritas na parte experimental e a Figura 2 mostra os cromatogramas sobrepostos dos padrões nos canais correspondentes. Observa-se que o tempo necessário para a eluição dos 29 pesticidas é de pouco mais de 7 min considerado, portanto, bastante rápido. Baseados nos tempos de retenção dos pesticidas, o método de aquisição foi dividido em 12 janelas de tempo para maximizar os sinais para os pesticidas.

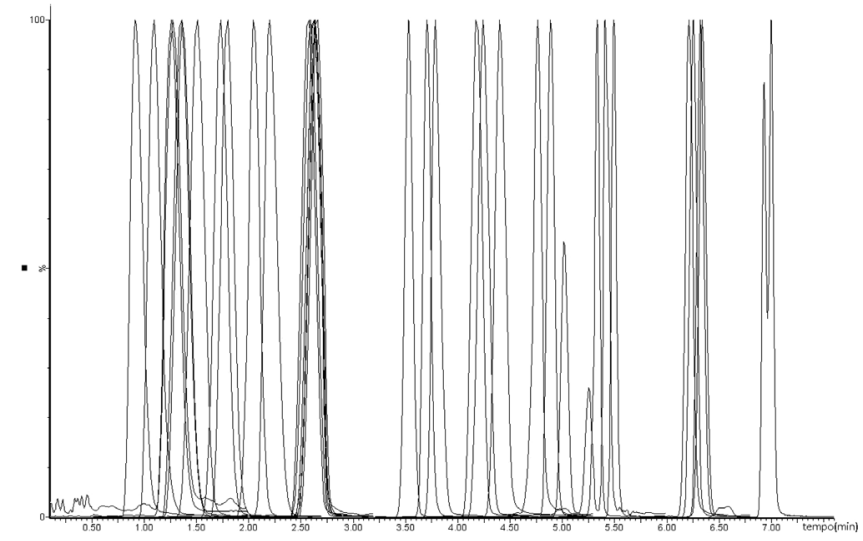

Figura 2. Cromatogramas sobrepostos dos padrões de pesticidas na concentração $0,1 \mu \mathrm{g} \mathrm{mL}^{-1}$. Sequência de eluição de acordo com os tempos de retenção apresentados na Tabela 1

Após a obtenção dos extratos em acetonitrila e posterior injeção de um volume de $10 \mu \mathrm{L}$ no sistema LC-MS/MS, observou-se a formação de picos não simétricos, causando problemas na quantificação de alguns analitos. Para solucionar este problema, a acetonitrila foi 
evaporada e o extrato seco foi ressuspenso em $2 \mathrm{~mL}$ de uma solução de $\mathrm{CH}_{3} \mathrm{OH} / \mathrm{H}_{2} \mathrm{O}$ com $0,1 \%$ de ácido fórmico (50\%, v/v), não havendo portanto pré-concentração. Este procedimento melhorou o formato dos picos, uma vez que a força do solvente da amostra ficou compatível com a da fase móvel.

\section{Validação do método}

O método foi validado separadamente para as matrizes alface, tomate, maçã e uva. Para isso determinaram-se os seguintes parâmetros de validação: linearidade; limite de detecção; limite de quantificação; seletividade; recuperação; repetitividade e precisão intermediária.

\section{Linearidade}

$\mathrm{O}$ uso de soluções de calibração na matriz tem sido frequentemente utilizado para contornar os problemas associados ao aumento ou supressão da ionização dos pesticidas nos sistemas cromatográficos acoplados a espectrômetros de massas. ${ }^{5} \mathrm{Um}$ dos potenciais problemas do efeito matriz é que diferentes amostras, tipos de extratos, commodities e diferentes "concentrações" da matriz podem apresentar efeitos desta natureza em diferentes graus de magnitude. ${ }^{27}$ Desse modo, como o propósito deste trabalho foi validar um método multirresíduo único para diferentes matrizes, baseado na extração por QuEChERS e UPLC-MS/MS, embora seja trabalhoso optou-se por utilizar a calibração na matriz, pois evita a obtenção de resultados inexatos.
Os coeficientes de determinação $\left(\mathrm{r}^{2}\right)$ obtidos foram maiores ou iguais a 0,99 e os CV (\%) foram menores do que $20 \%$ em todos os casos. O gráfico da função da calibração deve ser confeccionado e inspecionado visualmente, evitando-se a confiança cega nos coeficientes de correlação. ${ }^{27}$ Além disso, para assegurar que a calibração é adequada à região de interesse, para a detecção destes resíduos foram construídos os gráficos de resíduos e observou-se que não havia tendências.

Limite de quantificação (LOQ), exatidão e precisão

Também está apresentado nas Tabelas 2-5 o valor do limite de quantificação (LOQ) do método, que foi de $0,005 \mathrm{mg} \mathrm{kg}^{-1}$. Por definição, o LOQ é o nível mais baixo no qual tenha sido demonstrado que o critério para exatidão e precisão foi atingido. ${ }^{28}$

A precisão do método foi obtida através de estudo de repetitividade e precisão intermediária. As recuperações dos pesticidas foram testadas em triplicatas, em dois níveis de fortificação e em 2 ou 3 dias diferentes. As médias das recuperações, bem como os coeficientes de variação $(\mathrm{CV})$ de cada nível, estão apresentadas nas Tabelas 2-5. No documento do SANCO da Comunidade Europeia são estabelecidas as faixas de recuperação (70-120\%) bem como os coeficientes de variação $(\leq 20 \%)$ aceitáveis ${ }^{28}$ e neste trabalho foram utilizados estes critérios de aceitação.

As amostras foram fortificadas nos níveis de 0,005 e $0,010 \mathrm{mg}$ $\mathrm{kg}^{-1}$. Estes valores foram escolhidos com base no LOQ do método e também pelo fato de serem abaixo dos LMRs. A Comunidade

Tabela 2. Resultados da validação do alface

\begin{tabular}{|c|c|c|c|c|c|c|c|}
\hline \multirow[b]{2}{*}{$\mathrm{N}^{\circ}$} & \multirow[b]{2}{*}{ Composto } & \multirow{2}{*}{$\begin{array}{c}\mathrm{LMR}^{2} \\
\left(\mathrm{mg} \mathrm{kg}^{-1}\right)\end{array}$} & \multirow{2}{*}{$\begin{array}{c}\text { LOQ } \\
\left(\mathrm{mg} \mathrm{kg}^{-1}\right)\end{array}$} & \multicolumn{2}{|c|}{ Recuperação (\%) } & \multicolumn{2}{|c|}{ Coeficiente de Variação (\%) } \\
\hline & & & & $\begin{array}{c}0,005 \\
\left(\mathrm{mg} \mathrm{kg}^{-1}\right)\end{array}$ & $\begin{array}{c}0,010 \\
\left(\mathrm{mg} \mathrm{kg}^{-1}\right)\end{array}$ & $\begin{array}{c}0,005 \\
\left(\mathrm{mg} \mathrm{kg}^{-1}\right)\end{array}$ & $\begin{array}{c}0,010 \\
\left(\mathrm{mg} \mathrm{kg}^{-1}\right)\end{array}$ \\
\hline 1 & Tiabendazol & Não há & 0,005 & 80,1 & 79,4 & 10,9 & 3,5 \\
\hline 2 & Hexazinona & Não há & 0,005 & 97,5 & 97,5 & 10,5 & 4,6 \\
\hline 3 & Atrazina & Não há & 0,005 & 95,4 & 96,9 & 11,8 & 6,2 \\
\hline 4 & Pirimicarbe & 1,0 & 0,005 & 84,1 & 83,8 & 11,1 & 3 \\
\hline 5 & Metalaxil & Não há & 0,005 & 99,7 & 98,1 & 11,3 & 7,2 \\
\hline 6 & Diazinona & Não há & 0,005 & 84,4 & 83,1 & 1,5 & 9,3 \\
\hline 7 & Benalaxil & Não há & 0,005 & 95,8 & 93,3 & 12,7 & 9,9 \\
\hline 8 & Difenoconazol & 0,5 & 0,005 & 86,3 & 84,5 & 13,3 & 11,4 \\
\hline 9 & Imazalil & Não há & 0,005 & 88,0 & 86,6 & 14,0 & 9,5 \\
\hline 10 & Carbofurano & Não há & 0,005 & 100,2 & 101,0 & 12,7 & 1,6 \\
\hline 11 & cis-Mevinfós & 0,5 & 0,005 & 91,9 & 95,6 & 12,4 & 0,4 \\
\hline 12 & Clomazona & Não há & 0,005 & 96,4 & 100,1 & 13,6 & 12,1 \\
\hline 13 & Tebuconazol & Não há & 0,005 & 94,7 & 89,1 & 11,2 & 8,4 \\
\hline 14 & Malationa & Não há & 0,005 & 94,5 & 94,6 & 17,6 & 4,7 \\
\hline 15 & Fenarimol & Não há & 0,005 & 92,9 & 90,2 & 9,9 & 11,9 \\
\hline 16 & Azoxistrobina & 1,0 & 0,005 & 103,2 & 101,7 & 13,6 & 4,0 \\
\hline 17 & Diclorvos & Não há & 0,005 & 78,4 & 76,4 & 15,0 & 11,8 \\
\hline 18 & Dimetoato & Não há & 0,005 & 90,7 & 92,6 & 15,0 & 3,4 \\
\hline 19 & Simazina & Não há & 0,005 & 94,3 & 94,1 & 10,2 & 4,5 \\
\hline 20 & Ciproconazol & Não há & 0,005 & 95,3 & 94,8 & 10,7 & 5,6 \\
\hline 21 & Monocrotofós & Não há & 0,005 & 92,6 & 93,0 & 16,7 & 10,8 \\
\hline 22 & Metribuzin & Não há & 0,005 & 105,0 & 96,1 & 12,3 & 1,3 \\
\hline 23 & Aldicarbe & Não há & 0,005 & 85,7 & 93,3 & 14,6 & 2,3 \\
\hline 24 & Imidacloprido & 0,5 & 0,005 & 98,3 & 110,2 & 12,1 & 14,2 \\
\hline 25 & Bromacila & Não há & 0,005 & 91,2 & 94,1 & 9,7 & 5,7 \\
\hline 26 & Diuron & Não há & 0,005 & 89,0 & 97,1 & 18,3 & 15,9 \\
\hline 27 & Fentiona & Não há & 0,005 & 83,4 & 87,9 & 7,9 & 18,6 \\
\hline 28 & Procimidona & 5,0 & 0,005 & 109,0 & 83,4 & 7,7 & 19,5 \\
\hline 29 & Fludioxonil & Não há & 0,005 & 90,6 & 86,2 & 13,3 & 13,0 \\
\hline
\end{tabular}


Europeia estabelece um nível de $0,010 \mathrm{mg} \mathrm{kg}^{-1}$ para pesticidas não autorizados ou proibidos e para comida de bebês. ${ }^{30} \mathrm{Como}$ a aplicação do método é para monitorar pesticidas registrados e não registrados para as diversas culturas, escolheram-se limites de $0,010 \mathrm{mg} \mathrm{kg}^{-1} \mathrm{e}$ outro abaixo $\left(0,005 \mathrm{mg} \mathrm{kg}^{-1}\right)$. Além disso, os baixos níveis de fortificação demonstram a alta detectabilidade do instrumento UPLC-MS/ MS utilizado.

Observa-se na Tabela 2 que as recuperações e precisões obtidas para alface, para os dois níveis de fortificação para os 29 analitos variaram entre $79-110 \%$, com coeficiente de variação abaixo de $20 \%$, valores considerados aceitáveis. ${ }^{28}$

Na Tabela 3 estão apresentados os resultados da validação para a matriz tomate. Todos os resultados foram satisfatórios, exceto para o diclorvós.

Na Tabela 4 estão apresentados os valores para a maçã. Observa-se que para a maioria dos analitos os valores de recuperação e de precisão estão dentro da faixa aceitável, exceto para imazalil, cis-mevinfós, clomazona, diclorvós, aldicarbe e procimidona.

$\mathrm{Na}$ Tabela 5 estão apresentados os resultados da validação da uva. Os valores de recuperação e de precisão estão dentro da faixa aceitável para a maioria dos analitos, exceto para clomazona, tebuconazole, diclorvós e aldicarbe.

Os pesticidas diclorvós, clomazona, e aldicarbe foram considerados como sendo os mais problemáticos, pois apresentaram resultados insatisfatórios para 2 ou 3 das 4 commodities estudadas.
Robustez

O método validado mostrou ser robusto uma vez que pequenas alterações nas condições de extração (tempo de agitação, temperatura, entre outras) e cromatográficas (composição da fase móvel, temperatura da coluna, entre outras) não causaram diferenças significativas nos resultados.

\section{Participação de ensaio de proficiência}

Ensaio de proficiência (EP) é o uso de comparações interlaboratoriais com o objetivo de avaliar a habilidade de um laboratório em realizar um determinado ensaio ou medição de modo competente e demonstrar a confiabilidade dos resultados gerados. A fim de verificar a confiabilidade dos resultados obtidos utilizando o método validado, o Laboratório de Resíduos e Contaminantes (LRC) da Embrapa Meio Ambiente teve a oportunidade de participar do Ensaio de Proficiência (EP) para Determinação de Pesticidas em Alimentos $5^{\text {a }}$ Rodada Matriz Maçã, organizado pelo Instituto Nacional de Qualidade em Saúde - INCQS, unidade da Fundação Oswaldo Cruz (Fiocruz). ${ }^{31}$ A matriz recebida foi polpa de maçã que continha, dentre outros, o pesticida dimetoato. Através do método de ensaio descrito neste trabalho, obtiveram como avaliação de desempenho o valor do índice $\mathrm{Z} \leq 2$, ou seja, satisfatório de acordo com a ISO Guia 43-1 $1^{32}$ para este agrotóxico, corroborando a habilidade do método multirresíduo validado para esta substância.

Tabela 3. Resultados da validação do tomate

\begin{tabular}{|c|c|c|c|c|c|c|c|}
\hline \multirow[b]{2}{*}{$\mathrm{N}^{\circ}$} & \multirow[b]{2}{*}{ Composto } & \multirow{2}{*}{$\begin{array}{c}\mathrm{LMR}^{2} \\
\left(\mathrm{mg} \mathrm{kg}^{-1}\right)\end{array}$} & \multirow{2}{*}{$\begin{array}{c}\text { LOQ } \\
\left(\mathrm{mg} \mathrm{kg}^{-1}\right)\end{array}$} & \multicolumn{2}{|c|}{ Recuperação (\%) } & \multicolumn{2}{|c|}{ Coeficiente de Variação (\%) } \\
\hline & & & & $\begin{array}{c}0,005 \\
\left(\mathrm{mg} \mathrm{kg}^{-1}\right)\end{array}$ & $\begin{array}{c}0,010 \\
\left(\mathrm{mg} \mathrm{kg}^{-1}\right)\end{array}$ & $\begin{array}{c}0,005 \\
\left(\mathrm{mg} \mathrm{kg}^{-1}\right)\end{array}$ & $\begin{array}{c}0,010 \\
\left(\mathrm{mg} \mathrm{kg}^{-1}\right)\end{array}$ \\
\hline 1 & Tiabendazol & Não há & 0,005 & 80,7 & 78,8 & 11,4 & 5,1 \\
\hline 2 & Hexazinona & Não há & 0,005 & 78,6 & 79,1 & 7,4 & 6,9 \\
\hline 3 & Atrazina & Não há & 0,005 & 96,4 & 94,4 & 6,1 & 1,9 \\
\hline 4 & Pirimicarbe & 1,0 & 0,005 & 92,4 & 91,5 & 5,9 & 5,3 \\
\hline 5 & Metalaxil & 0,05 & 0,005 & 98,2 & 99,8 & 5,6 & 4,3 \\
\hline 6 & Diazinona & Não há & 0,005 & 86,1 & 87,6 & 11,7 & 8,4 \\
\hline 7 & Benalaxil & 0,5 & 0,005 & 96,1 & 95,4 & 8,6 & 4,2 \\
\hline 8 & Difenoconazol & 0,1 & 0,005 & 93,7 & 92,9 & 1,3 & 4,7 \\
\hline 9 & Imazalil & Não há & 0,005 & 86,1 & 89,3 & 11,4 & 4,7 \\
\hline 10 & Carbofurano & 0,1 & 0,005 & 80,3 & 76,9 & 5,7 & 4,6 \\
\hline 11 & cis-Mevinfós & 0,2 & 0,005 & 83,2 & 87,7 & 15,4 & 12,1 \\
\hline 12 & Clomazona & Não há & 0,005 & 85,8 & 86,9 & 7,5 & 6,2 \\
\hline 13 & Tebuconazol & 0,1 & 0,005 & 94,9 & 93,3 & 7,4 & 9,2 \\
\hline 14 & Malationa & 3,0 & 0,005 & 90,9 & 95,5 & 8,4 & 6,0 \\
\hline 15 & Fenarimol & Não há & 0,005 & 99,0 & 93,1 & 3,6 & 5,4 \\
\hline 16 & Azoxistrobina & 0,5 & 0,005 & 98,8 & 101,0 & 11,3 & 8,7 \\
\hline 17 & Diclorvos & Não há & 0,005 & 44,8 & 64,4 & 54,6 & 34,5 \\
\hline 18 & Dimetoato & 1,0 & 0,005 & 94,2 & 95,5 & 6,0 & 4,7 \\
\hline 19 & Simazina & Não há & 0,005 & 80,6 & 74,6 & 8,2 & 8,4 \\
\hline 20 & Ciproconazol & Não há & 0,005 & 83,5 & 97,9 & 10,2 & 5,8 \\
\hline 21 & Monocrotofós & Não há & 0,005 & 87,1 & 86,3 & 11,9 & 6,6 \\
\hline 22 & Metribuzin & 0,1 & 0,005 & 85,4 & 84,5 & 16,2 & 9,6 \\
\hline 23 & Aldicarbe & Não há & 0,005 & 90,7 & 87,1 & 15,7 & 8,9 \\
\hline 24 & Imidacloprido & 0,5 & 0,005 & 113,9 & 102,8 & 6,7 & 2,5 \\
\hline 25 & Bromacila & Não há & 0,005 & 71,0 & 71,5 & 8,2 & 4,6 \\
\hline 26 & Diuron & Não há & 0,005 & 96,8 & 95,5 & 8,6 & 5,6 \\
\hline 27 & Fentiona & Não há & 0,005 & 83,3 & 80,5 & 11,4 & 4,7 \\
\hline 28 & Procimidona & 2,0 & 0,005 & 89,5 & 96,6 & 14,2 & 4,8 \\
\hline 29 & Fludioxonil & Não há & 0,005 & 76,2 & 86,9 & 10,8 & 7,9 \\
\hline
\end{tabular}


Tabela 4. Resultados da validação da maçã

\begin{tabular}{|c|c|c|c|c|c|c|c|}
\hline \multirow[b]{2}{*}{$\mathrm{N}^{\circ}$} & \multirow[b]{2}{*}{ Composto } & \multirow{2}{*}{$\begin{array}{c}\mathrm{LMR}^{2} \\
\left(\mathrm{mg} \mathrm{kg}^{-1}\right)\end{array}$} & \multirow[b]{2}{*}{$\begin{array}{c}\text { LOQ } \\
\left(\mathrm{mg} \mathrm{kg}^{-1}\right)\end{array}$} & \multicolumn{2}{|c|}{ Recuperação (\%) } & \multicolumn{2}{|c|}{ Coeficiente de Variação (\%) } \\
\hline & & & & $\begin{array}{c}0,005 \\
\left(\mathrm{mg} \mathrm{kg}^{-1}\right)\end{array}$ & $\begin{array}{c}0,010 \\
\left(\mathrm{mg} \mathrm{kg}^{-1}\right)\end{array}$ & $\begin{array}{c}0,005 \\
\left(\mathrm{mg} \mathrm{kg}^{-1}\right)\end{array}$ & $\begin{array}{c}0,010 \\
\left(\mathrm{mg} \mathrm{kg}^{-1}\right)\end{array}$ \\
\hline 1 & Tiabendazol & 10,0 & 0,005 & 74,4 & 81,7 & 16,0 & 5,6 \\
\hline 2 & Hexazinona & Não há & 0,005 & 97,7 & 95,7 & 4,0 & 1,9 \\
\hline 3 & Atrazina & Não há & 0,005 & 100,3 & 99,2 & 5,2 & 4,1 \\
\hline 4 & Pirimicarbe & Não há & 0,005 & 95,6 & 95,8 & 5,4 & 3,2 \\
\hline 5 & Metalaxil & 1,0 & 0,005 & 95,9 & 95,0 & 5,8 & 3,8 \\
\hline 6 & Diazinona & 0,5 & 0,005 & 81,2 & 84,4 & 18,3 & 11,0 \\
\hline 7 & Benalaxil & 0,2 & 0,005 & 96,6 & 95,2 & 2,6 & 3,0 \\
\hline 8 & Difenoconazol & 0,5 & 0,005 & 96,1 & 98,7 & 4,9 & 3,7 \\
\hline 9 & Imazalil & 2,0 & 0,005 & 68,3 & 64,5 & 8,3 & 14,7 \\
\hline 10 & Carbofurano & Não há & 0,005 & 96,4 & 94,5 & 7,9 & 6,0 \\
\hline 11 & cis-Mevinfós & Não há & 0,005 & 58,6 & 63,5 & 48,3 & 36,0 \\
\hline 12 & Clomazona & Não há & 0,005 & 134,0 & 89,8 & 71,4 & 11,5 \\
\hline 13 & Tebuconazol & 0,1 & 0,005 & 105,9 & 96,6 & 6,3 & 5,7 \\
\hline 14 & Malationa & 2,0 & 0,005 & 100,8 & 96,7 & 8,9 & 6,2 \\
\hline 15 & Fenarimol & 0,05 & 0,005 & 98,7 & 96,0 & 9,5 & 8,4 \\
\hline 16 & Azoxistrobina & Não há & 0,005 & 99,0 & 98,3 & 6,1 & 3,3 \\
\hline 17 & Diclorvos & Não há & 0,005 & 27,7 & 12,1 & 96,7 & 125,7 \\
\hline 18 & Dimetoato & 2,0 & 0,005 & 96,4 & 95,9 & 11,3 & 5,8 \\
\hline 19 & Simazina & 0,02 & 0,005 & 95,5 & 99,4 & 8,1 & 4,9 \\
\hline 20 & Ciproconazol & 0,4 & 0,005 & 105,9 & 101,2 & 6,3 & 6,0 \\
\hline 21 & Monocrotofós & Não há & 0,005 & 93,0 & 89,8 & 8,6 & 5,4 \\
\hline 22 & Metribuzin & Não há & 0,005 & 99,4 & 104,1 & 9,6 & 7,9 \\
\hline 23 & Aldicarbe & Não há & 0,005 & 41,5 & 61,3 & 66,2 & 40,9 \\
\hline 24 & Imidacloprido & Não há & 0,005 & 97,1 & 96,8 & 10,1 & 5,5 \\
\hline 25 & Bromacila & Não há & 0,005 & 94,5 & 95,7 & 12,7 & 9,9 \\
\hline 26 & Diuron & Não há & 0,005 & 108,9 & 104,2 & 9,2 & 8,7 \\
\hline 27 & Fentiona & 1,0 & 0,005 & 81,9 & 89,8 & 37,1 & 13,9 \\
\hline 28 & Procimidona & 2,0 & 0,005 & 121,8 & 83,1 & 47,0 & 36,5 \\
\hline 29 & Fludioxonil & Não há & 0,005 & 112,5 & 95,9 & 21,3 & 13,0 \\
\hline
\end{tabular}

Tabela 5. Resultados da validação da uva

\begin{tabular}{|c|c|c|c|c|c|c|c|}
\hline \multirow[b]{2}{*}{$\mathrm{N}^{\circ}$} & \multirow[b]{2}{*}{ Composto } & \multirow{2}{*}{$\begin{array}{c}\mathrm{LMR}^{2} \\
\left(\mathrm{mg} \mathrm{kg}^{-1}\right)\end{array}$} & \multirow{2}{*}{$\begin{array}{c}\text { LOQ } \\
\left(\mathrm{mg} \mathrm{kg}^{-1}\right)\end{array}$} & \multicolumn{2}{|c|}{ Recuperação (\%) } & \multicolumn{2}{|c|}{ Coeficiente de Variação (\%) } \\
\hline & & & & $\begin{array}{c}0,005 \\
\left(\mathrm{mg} \mathrm{kg}^{-1}\right)\end{array}$ & $\begin{array}{c}0,010 \\
\left(\mathrm{mg} \mathrm{kg}^{-1}\right)\end{array}$ & $\begin{array}{c}0,005 \\
\left(\mathrm{mg} \mathrm{kg}^{-1}\right)\end{array}$ & $\begin{array}{c}0,010 \\
\left(\mathrm{mg} \mathrm{kg}^{-1}\right)\end{array}$ \\
\hline 1 & Tiabendazol & Não há & 0,005 & 87,1 & 84,7 & 2,8 & 13,1 \\
\hline 2 & Hexazinona & Não há & 0,005 & 107,3 & 106,2 & 0,7 & 0,1 \\
\hline 3 & Atrazina & Não há & 0,005 & 107,8 & 103,9 & 1,5 & 6,2 \\
\hline 4 & Pirimicarbe & 1,0 & 0,005 & 101,0 & 97,9 & 0,7 & 4,2 \\
\hline 5 & Metalaxil & 1,0 & 0,005 & 108,7 & 104,2 & 2,2 & 5,1 \\
\hline 6 & Diazinona & Não há & 0,005 & 100,8 & 99,5 & 2,5 & 0,1 \\
\hline 7 & Benalaxil & 0,2 & 0,005 & 109,0 & 108,8 & 0,7 & 1,4 \\
\hline 8 & Difenoconazol & 0,2 & 0,005 & 105,8 & 102,6 & 1,0 & 2,3 \\
\hline 9 & Imazalil & Não há & 0,005 & 78,0 & 71,9 & 8,4 & 21,0 \\
\hline 10 & Carbofurano & Não há & 0,005 & 111,5 & 111,4 & 0,3 & 4,8 \\
\hline 11 & cis-Mevinfós & Não há & 0,005 & 79,5 & 82,1 & 20,2 & 6,5 \\
\hline 12 & Clomazona & Não há & 0,005 & 145,4 & 104,5 & 44,5 & 9,3 \\
\hline 13 & Tebuconazol & 2,0 & 0,005 & 59,0 & 90,9 & 11,1 & 3,1 \\
\hline 14 & Malationa & Não há & 0,005 & 103,7 & 101,4 & 2,4 & 0,3 \\
\hline 15 & Fenarimol & 0,05 & 0,005 & 108,7 & 101,0 & 4,4 & 2,0 \\
\hline 16 & Azoxistrobina & 0,5 & 0,005 & 110,6 & 105,0 & 0,4 & 2,4 \\
\hline 17 & Diclorvos & Não há & 0,005 & 24,7 & 21,1 & 94,5 & 21,7 \\
\hline 18 & Dimetoato & Não há & 0,005 & 108,2 & 101,7 & 1,6 & 3,1 \\
\hline 19 & Simazina & 0,02 & 0,005 & 100,4 & 96,6 & 0,5 & 6,3 \\
\hline 20 & Ciproconazol & 0,1 & 0,005 & 103,4 & 102,6 & 9,9 & 6,6 \\
\hline 21 & Monocrotofós & Não há & 0,005 & 102,5 & 99,2 & 2,6 & 4,0 \\
\hline 22 & Metribuzin & Não há & 0,005 & 108,1 & 101,5 & 5,1 & 13,1 \\
\hline 23 & Aldicarbe & Não há & 0,005 & 70,6 & 58,2 & 2,5 & 20,7 \\
\hline 24 & Imidacloprido & 1,0 & 0,005 & 107,9 & 105,5 & 4,5 & 4,3 \\
\hline 25 & Bromacila & Não há & 0,005 & 104,5 & 102,0 & 2,4 & 14,2 \\
\hline 26 & Diuron & 0,1 & 0,005 & 104,4 & 99,3 & 7,0 & 15,2 \\
\hline 27 & Fentiona & 0,5 & 0,005 & 93,7 & 87,2 & 0,8 & 15,6 \\
\hline 28 & Procimidona & 5,0 & 0,005 & 104,3 & 85,9 & 12,7 & 16,8 \\
\hline 29 & Fludioxonil & Não há & 0,005 & 117,6 & 98,6 & 2,4 & 17,5 \\
\hline
\end{tabular}




\section{Avaliação de amostras comerciais}

Após a validação do método multirresíduo, o mesmo foi utilizado para análises de amostras reais. Dez amostras de cada matriz foram adquiridas de estabelecimentos comerciais de Jaguariúna e Campinas, ambas localizadas no estado de São Paulo, e encaminhadas ao laboratório, onde foram processadas e armazenadas em freezer $\left(-18^{\circ}\right.$ C) até o momento das análises.

Nas análises de alface foi detectada a presença de metalaxil em uma das amostras, sendo que este pesticida não possui registro para esta cultura. Imidacloprido foi detectado em 6 das 10 amostras analisadas, sendo que em 2 os valores estavam acima do LMR $(0,5$ $\left.\mathrm{mg} \mathrm{kg}^{-1}\right)$. Procimidona foi detectada em 3 amostras, porém abaixo do $\operatorname{LMR}\left(2,0 \mathrm{mg} \mathrm{kg}^{-1}\right)$.

Nas amostras de tomate foi detectada a presença de difenoconazol (LMR 0,1 $\mathrm{mg} \mathrm{kg}^{-1}$ ) e procimidona (LMR 2,0 $\mathrm{mg} \mathrm{kg}^{-1}$ ) em níveis abaixo do permitido. Imidacloprido foi detectado em 7 amostras, sendo que todas estavam abaixo do $\operatorname{LMR}\left(0,5 \mathrm{mg} \mathrm{kg}^{-1}\right)$.

Nas amostras de maçã foram detectados os seguintes pesticidas: difenoconazol (LMR 0,5 mg kg-1), malationa (LMR 2,0 mg kg-1) e dimetoato (LMR 2,0 $\mathrm{mg} \mathrm{kg}^{-1}$ ) em níveis abaixo dos permitidos.

Nas amostras de uva foram detectados difenoconazole (LMR 0,2 $\mathrm{mg} \mathrm{kg}^{-1}$ ), tebuconazole (LMR 2,0 $\mathrm{mg} \mathrm{kg}^{-1}$ ), imidacloprido (LMR 1,0 $\mathrm{mg} \mathrm{kg}^{-1}$ ), todos abaixo do LMR. Foi detectado fludioxonil em 4 amostras, sendo que este pesticida não possui registro para esta cultura.

Análises de amostras reais foram realizadas e os resultados confirmam a necessidade de realizar monitoramento de resíduos de pesticidas nos alimentos.

\section{CONCLUSÃO}

Neste estudo foi utilizado o procedimento do método QuEChERS no preparo das amostras, o qual permitiu a extração de multirresíduo de pesticidas nas matrizes tomate, alface, maçã e uva e posterior confirmação e quantificação por UPLC-MS/MS. O método apresentou bons resultados de linearidade para os 29 compostos e permitiu a determinação de limites de quantificação abaixo dos LMRs com precisões e exatidões dentro das faixas aceitáveis para a maioria dos pesticidas. O método validado mostrou ser rápido, eficiente e confiável e pode ser utilizado no monitoramento de resíduos de pesticidas nas matrizes selecionadas.

\section{REFERÊNCIAS}

1. Camino-Sánchez, F. J.; Zafra-Gómez, A.; Oliver-Rodriguez, B.; Ballesteros, O.; Navalón, A.; Crovetto, G.; Vilchez, J. L.; Food Addit. Contam. 2010, 27, 1532.

2. http://portal.anvisa.gov.br/wps/portal/anvisa/home/ agrotoxicotoxicologia, acessada em Julho 2011.

3. Cajka, T.; Hajslova, J.; Lacina, O.; Mastovska, K.; Lehotay, S. J.; J. Chromatogr. A 2008, 1186, 281.

4. Pihlstrom, T.; Blomkvist, G.; Friman, P.; Pagard, U.; Osterdahl, B.; Anal. Bioanal. Chem. 2007, 389, 1773.

5. Lehotay, S. J.; Mastovska, K.; Lightfield, A. R.; Gates, R. A.; J. AOAC Int. 2010, 93, 355.

6. Lacina, O.; Urbanova, J.; Poustka, J.; Hajslova, J.; J. Chromatogr. A 2010, 1217, 648 .
7. Soler, C.; Picó, Y.; Trends Anal. Chem. 2007, 26, 103.

8. Lehotay, S. J.; Anastassiades, M.; Majors, R. E.; LC-GC Europe 2010, 8,418 .

9. Anastassiades, M.; Lehotay, S. J.; Stajnbaher, D.; Schenck, F. J.; J. AOAC Int. 2003, 86, 412 .

10. Prestes, O. D.; Friggi, C. A.; Adaime, M. B.; Zanella, R.; Quim. Nova 2009, 32, 1620.

11. Maldaner, L.; Jardim, I. C. S. F.; Quim. Nova 2009, 32, 214.

12. Yu, K.; Little, D.; Plumb, R.; Smith, B.; Rapid Commun. Mass Spectrom. 2006, 20, 544.

13. Frenich, A. G.; Martínez Vidal, J. L.; Pastor-Montoro, E.; RomeroGonzález, R.; Anal. Bioanal. Chem. 2008, 390, 947.

14. Romero-González, R.; Frenich, A. G.; Vidal, J. L. M.; Talanta 2008, 76, 211.

15. Chan, E. C. Y.; Yap, S. L.; Lau, A. J.; Leow, P. C.; Toh, D. F.; Koh, H. L.; Rapid Commun. Mass Spectrom. 2007, 21, 519.

16. Chesnut, S. M.; Salisbury, J. J.; J. Sep. Sci. 2007, 30, 1183.

17. Kasprzyk-Hordern, B.; Dinsdale, R. M.; Guwy, A. J.; J. Chromatogr., A 2007, 1161, 132.

18. Leandro, C. C.; Hancock, P.; Fussell, R. J.; Keely, B. J.; J. Chromatogr, A 2007, 1144, 161.

19. Mastovska, K.; Dorweiler, K. J.; Lehotay, S. J.; Wegscheid, J. S.; Szpylka, K. A.; J. Agric. Food Chem. 2010, 58, 5959.

20. Zhang, K.; Wong, J. W.; Hayward, D. G.; Sheladia, P.; Krynitsky, A. J.; Schenck, F. J.; Webster, M. G.; Ammann, J. A.; Ebeler, S. E.; J. Agric. Food Chem. 2009, 57, 4019.

21. Fernández, R.; Garrido Frenich, A.; Martínez Vidal, J. L.; Romero González, R.; Hernández Torres, M. E.; Anal. Sci. 2009, 25, 535.

22. Chen. G.; Cao, P.; Liu, R.; Food Chem. 2011, 125, 1406.

23. Kovalczuk, T.; Lacina, O.; Jech, M.; Poustka, J.; Hajslova, J.; Food Addit. Contam. 2008, 25, 444.

24. Zhang, K.; Wong, J. W.; Hayward, D. G.; Sheladia, P.; Krynitsky, A. J.; Schenck, J.; Webster, G.; Ammann, J. A.; Ebeler, S.; J. Agric. Food. Chem. 2009, 57, 4019.

25. Jardim, I. C. S. F.; Andrade, J. A. A.; Queiroz, S. C. N.; Quim. Nova 2009, 32, 996.

26. Cardoso, M. H. W. M.; Gouvêa, A. V.; Nóbrega, A. W.; Abrantes, S. M. P.; Ciência Tecnol. Alim. 2010, 30, 63.

27. www.anvisa.gov.br/reblas/para/CONTROLE_QUALIDADE.pdf, acessada em Julho 2011.

28. SANCO/10684/2009; Method Validation and Quality Control Procedures for Pesticide Residues Analysis in Food And Feed, implemented by 01/01/2010.

29. European Committee for Standardization-CEN; CEN/TC 275 15662:2008: Foods of plant origin - Determination of pesticide residues using GC-MS and/or LC-MS/MS following acetonitrile extraction/ partitioning and clean-up by dispersive SPE - QuEChERS-method, European Union, 2008.

30. Wang, I.; Leung. D.; J. Agric. Food. Chem. 2009, 57, 2162.

31. http://www.incqs.fiocruz.br/images/stories/incqs/ensaio/EP_Relatorio_ AGR_05_10.pdf, acessada em Julho 2011.

32. Associação Brasileira de Normas Técnicas - ABNT; ABNT ISO/ IEC Guia 43-1: 1999. Ensaio de Proficiência por comparações interlaboratoriais - Parte 1. Desenvolvimento e Operação de programas de Ensaios de Proficiência, ABNT: Rio de Janeiro, 1999. 